\title{
The future of the NHS: the state of care in hospitals
}

\author{
Emeritus Professor Alan Glasper, from the University of Southampton, discusses the Care Quality \\ Commission's annual report looking at health and social care in England
}

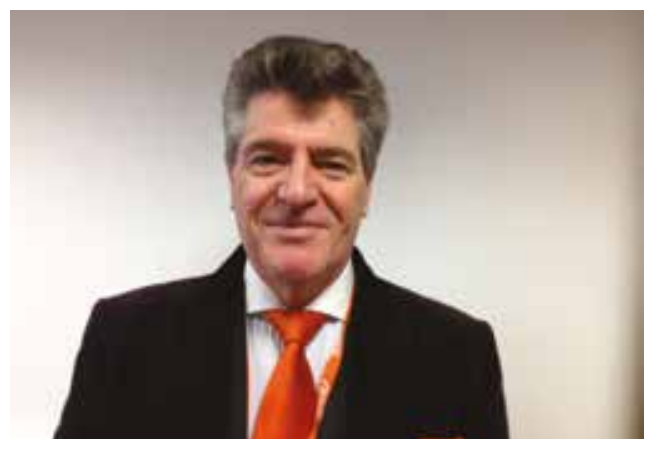

I n late November 2016 the Care Quality Commission (CQC) published its annual appraisal of health and social care delivery in England. Named The State of Health Care and Adult Social Care in England 2015/16, the report shows that many health and care services in England were providing good quality care, despite challenging environments, but that substantial variation remained (CQC, 2016a). NHS hospital care will be discussed in this column. The CQC report (2016a: 13) states that:

- People are living longer, and many older people are living with multiple complex and long-term conditions

- There is an increasing demand for adult social care and high levels of unmet need among older people

- Nearly $66 \%$ of NHS providers recorded a deficit (the majority were acute hospital trusts)

- There is a shortage of GPs combined with increasing vacancy levels in primary care

- Some providers are starting to shift towards new models of delivering care, for example by using new technologies such as telemedicine (Glasper, 2016).

The report outlines that NHS hospitals in England are under increasing pressure, which is threatening their ability to deliver optimum care to patients. Despite this, many hospital core services were rated good or outstanding by the CQC, especially services for children and young people. However, the report also reveals that some core services such as urgent and emergency services and medical care need to improve. This has become exacerbated throughout the first week of the New Year with new headlines on the British Red Cross' assertions of a 'humanitarian crisis' in NHS hospitals, especially emergency departments (Campbell et al, 2017)

\section{Background}

The CQC is the independent regulator of health and social care in England. Its primary role is to ensure that health and social care services provide people with safe, effective, compassionate, and responsive high-quality care, which is well led by professionals who put people at the heart of their activities. These activities have become more focused and reinforced since the events that took place at the Mid Staffordshire NHS Foundation Trust (Francis, 2013). Patients who should have received high standards of care were badly let down by a hospital that should have known better. Subsequent to the findings in the report (Francis, 2013), the CQC developed fundamental standards for healthcare providers such as hospitals. These fundamental standards are the standards below which care in institutions must never fall. The CQC conducts inspections to monitor how these fundamental standards of care are applied in practice throughout the health service in England.

The CQC insists that service users have the right to expect the following standards

- Person-centred care

- Dignity and respect

- Informed consent must be obtained from service users

- Protection against harm

- Protection against abuse

- Provided with sufficient food and drink to maintain health

- The care environment and equipment used is clean, suitable and appropriately maintained, stored and used correctly.

- To be freely able to raise complaints without fear of retribution

- Care providers must be open and transparent about care and treatment and service users should expect that the duty of candour is always applied

- Care institutions must operate under good governance and formulate plans to ensure that the CQC's fundamental standards are met

- Healthcare providers must ensure that they have sufficient suitably qualified, competent and experienced staff

- Healthcare providers must employ fit and proper staff who can provide care and treatment appropriate to their role

- All providers of care must display their CQC ratings in prominent places. (CQC, 2016b)

\section{Ensuring compliance} to the fundamental standards

The CQC, in the aftermath of the Francis Report, developed more robust procedures for inspecting hospitals. Led by Professor Sir Mike Richards, the Chief Inspector of Hospitals, the CQC now carries out inspection within five key domains namely:

- Safety

- Effectiveness

- Caring

- Responsiveness

- Well-led (CQC, 2016c)

Richards and his team have formulated a range of key lines of enquiry for each of these domains, which help CQC inspectors and their specialist advisors (doctors, nurses and other health professionals) to make accurate judgements as to how each core service within a hospital meets the fundamental standards of care. Each core service is rated on each domain from outstanding, good, or requires improvement through to inadequate.

Through the new inspection methodology 
the CQC has completed the first full programme of hospital inspections and has awarded them ratings. The regulator now has a substantial baseline from which to draw conclusions about the quality and safety of care and what influences this within the hospital sector. In addition to other health and social care services the CQC inspected 133 NHS acute hospital trusts and 35 independent acute hospitals.

\section{CQC assessment of hospital care}

The report shows that $51 \%$ of NHS acute hospital trusts' core services that were inspected were rated as good, with $5 \%$ rated as outstanding. A total of $76 \%$ of services that had been judged previously as inadequate were found to have improved on subsequent inspections, thus reinforcing the value of the CQC inspections.

A team from Manchester Business School and The King's Fund were commissioned to evaluate the new CQC approach to hospital inspections and found that hospital staff were, on the whole, supportive of the new inspection methodology, perceiving it to be more credible, rigorous and in-depth, and much less likely to miss any significant issues compared with the old system (Walshe et al, 2014).

Despite concerns that some hospital are failing, this annual CQC report shows that there is a great deal to celebrate in the NHS especially in the way that many hospital staff, managers and leaders consistently — and often in the face of adversity - continue to deliver high-quality holistic care.

Although the CQC report indicates that adult social care services have been able to maintain quality throughout the inspection period, there are worrying indications that the sustainability of adult social care is approaching a tipping point. This in turn will impact on the ability of the acute care hospitals to maintain optimum levels of service delivery. There has been an increased demand during 2015/16 on core services such as emergency departments, exacerbated by failures in adult social care burdened by an increasing population of older people.

\section{'Bed occupancy rates exceeded $91 \%$ in January to March 2016; the highest quarterly rate for at least 6 years.' CQC, 2016a:9}

Furthermore, there has been an increase in the number of older people having to wait to be discharged from hospital, which reflects, in many cases, a lack of suitable care options after discharge. The future of the NHS may well be in danger unless changes are made to the way in which society cares for older people. As revealed by the British Red Cross, in the early weeks of January 2017 no less than 14 health trusts warned of overcrowding in their emergency departments with a corresponding negative impact on the efficiency of ambulance services (British Red Cross, 2017).

The British Red Cross believed that much of this was caused by an inability of hospitals to discharge people and this was attributed to a lack of the right level of care and support being available to them in the community. A lack of investment in care services for older and infirm adults, worsened by cuts to funding coupled with an increasing demand, have led to serious deficits in care provision. This has resulted in some older people being inappropriately cared for as inpatients when in fact they were fit enough to go home, if only the right support were available at home. This blocks inpatient beds that are needed for new patients, or in the worst case scenario service users are discharged without adequate care and treatment support.

In context, the CQC report shows that there were marked difference between the proportion of services for children and young people rated as good (63\%) and the proportion of urgent and emergency services rated as good (38\%). This suggests that service users can potentially experience different levels of care quality depending on the core services they need to use.

\section{Discussion}

The situation in emergency departments, coupled with an inability to effectively manage elective admissions compounded by severe financial conditions, has put intense pressure on NHS hospitals. It is important to remember that the CQC report shows that inspectors observed that the majority of staff treated their patients with respect and dignity. They saw that patient's privacy was upheld and that staff worked hard to explain to patients and carers what their care involved.

An ongoing challenge for hospitals in 2017 will be to ensure that safe care is consistently delivered. The CQC supports the Five Year Forward View (NHS England, 2014) which set out plans to develop new care models that aim to improve the quality of care and efficiency through the integration of services.

\section{KEY POINTS}

- The Care Quality Commission's The State of Health Care and Adult Social Care in England 2015/16 annual report was published in November 2016

- The report shows that NHS hospitals in England are under increasing pressure, which is threatening their ability to deliver optimum care to patients.

- The CQC developed fundamental standards below which care in institutions must never fall

- The report shows that $51 \%$ of NHS acute hospital trusts' core services that were inspected were rated as good, with $5 \%$ rated as outstanding.

- The worrying situation in emergency departments has put intense pressure on the NHS hospitals

There is no doubt that NHS trusts are faced with real challenges in 2017 and beyond that are set to continue, as hospitals face increasing demands on their services and deal with ongoing financial pressures. However the CQC annual report is tinged with a degree of optimism for the NHS. The CQC has made a commitment to work with its partners to offer the NHS whatever support it can to make the changes necessary to ensure highquality care into the future. Much of this will fall on the members of the nursing profession whose commitment and loyalty to the NHS is beyond reproach. BJN

British Red Cross (2017) Red Cross calls on government to allocate funds for health and social care. 6 January. http:// tinyurl.com/zslg65p (accessed 16 January 2017)

Campbell D, Morris S, Marsh S (2017) NHS faces

'humanitarian crisis' as demand rises, British Red Cross warns. Guardian. 6 January. http://tinyurl.com/h8dw94k (accessed 16 January 2017)

Care Quality Commission (2016a) The State of Health Care and Adult Social Care in England. http://tinyurl.com/ jjdy7ez (accessed 16 January 2017)

Care Quality Commission (2016b) The fundamental standards. http://tinyurl.com/jmgryq8 (accessed 17 January 2017)

Care Quality Commission (2016c) The five key questions we ask. http://tinyurl.com/jxwuffm (accessed 17 January 2017)

Francis R (2013) Report of the Mid Staffordshire NHS Foundation Trust Public Inquiry. http://tinyurl.com/ p2ebw82 (accessed 16 January 2017)

Glasper A (2016) Expanding the use of digital technology across the NHS. Br J Nurs 25(19): 984-5

NHS England (2014) Five Year Forward View. http://tinyurl. com/oxq92je (accessed 16 January 2017)

Walshe K, Addicott R, Boyd A, Robertson R, Ross S (2014) Evaluating the Care Quality Commission's acute hospital regulatory model: final report. http://tinyurl.com/ pzqugom (accessed 17 January 2017) 